\title{
A Single Scalar Field Model of Dark Energy with Equation of State Crossing -1
}

\author{
Mingzhe L* \\ Institut für Theoretische Physik, Philosophenweg 16, 69120 Heidelberg, Germany \\ Bo Fend and Xinmin Zhand \\ Institute of High Energy Physics, Chinese Academy of Sciences, \\ P.O. Box 918-4, Beijing 100049, People's Republic of China
}

\begin{abstract}
In this paper we study the possibility of building models of dark energy with equation of state across -1 and propose explicitly a model with a single scalar field which gives rise to an equation of state larger than -1 in the past and less than -1 at the present time, consistent with the current observations.
\end{abstract}

PACS number: 98.80.Cq

Since two groups 1, 2] independently showed the accelerating expansion of our universe based on the Type Ia Supernova (SN) observations of the redshift-distance relations in 1998, numerous observations and experiments consistently indicate that our universe is dominated by a component with a negative pressure, dubbed dark energy in the literature. Especially the first year Wilkinson Microwave Anisotropy Probe (WMAP) data [3] strongly support for a concordance model in which about $73 \%$ of the energy of the universe is indwelt in the dark energy ingredient. One essential to understand the nature of the dark energy is to detect the evolution of its equation of state $w=p / \rho$, where $p$ and $\rho$ are the pressure and the energy density of the dark energy respectively. Recent analysis on the data from the Supernova, cosmic microwave background (CMB) and large scale structure (LSS) show that the cosmological constant fits well to the data [4], however current data also mildly favor an evolving dark energy with an equation of state $w$ larger than -1 in the past and less than -1 today [5, 6] and evolving across -1 in the intermediate redshift. If such a result holds on with the accumulation of observational data, this would be a great challenge to the current models of dark energy. Firstly, the cosmological constant as a candidate for dark energy will be excluded and dark energy must be dynamical. Secondly, the simple dynamical dark energy models considered vastly in the literature like the quintessence or the phantom can not be satisfied either.

In the quintessence models 7, 8, 9, 10, $w$ is evolving however always in the range of $-1 \leq w \leq 1$. In the phantom models [11], one has $w \leq-1$. For the general k-essence models $\mathcal{L}=p(\phi, X)$ with $X=1 / 2 \nabla_{\mu} \phi \nabla^{\mu} \phi$, and $\nabla_{\mu}$ the covariant derivative, it was shown that it also fails to cross the boundary of $w=-1$ because of the problems of singularity and classical instability in the perturbative equations [12, 13]. Thus it is interesting to

\footnotetext{
*Electronic address: m.li@thphys.uni-heidelberg.de

${ }^{\dagger}$ Electronic address: fengbo@mail.ihep.ac.cn

‡Electronic address: xmzhang@mail.ihep.ac.cn
}

ask if theoretically there exists model of a single scalar field which has a $w$ crossing -1 during the evolution of the universe ${ }^{1}$. In this paper we propose a toy model of this type.

In the general k-essence models, the Lagrangian is a function of the field $\phi$ and its first derivative $\nabla_{\mu} \phi$. In this paper we will propose a dark energy model which includes higher derivative operators in the Lagrangian, for example,

$$
\mathcal{L}=\mathcal{L}\left(\phi, X, \square \phi \square \phi, \nabla_{\mu} \nabla_{\nu} \phi \nabla^{\mu} \nabla^{\nu} \phi\right),
$$

where $\square \equiv \nabla_{\mu} \nabla^{\mu}$ is the d'Alembertian operator. The higher derivative terms in the effective Lagrangian above may be derived from fundamental theories. In fact it has been shown in the literature that this type of operators does appear as the quantum corrections or due to the non-local physics in the string theory [24, 25, 26]. With the higher derivative terms to the Einstein gravity, the theory is shown to be renormalizable [27] which has attracted many attentions. Recently, higher derivative operators have been considered to stabilize the linear fluctuations in the scenario of "ghost condensation" 28]. In addition the canonical form for the higher derivative theory has been put forward by Ostrogradski about one and a half century ago 29. In short, it is interesting and worthwhile to study the implications of models with higher derivatives in cosmology.

In this paper we will show this type of theories provides a class of models of dark energy where the equation of state can get across -1 during evolution, consistent with the current observations. We consider the simple model with the Lagrangian given by ${ }^{2}$

$$
\mathcal{L}=-\frac{1}{2} \nabla_{\mu} \phi \nabla^{\mu} \phi+\frac{c}{2 M^{2}} \square \phi \square \phi-V(\phi),
$$

\footnotetext{
1 The dark energy models with the property of crossing the cosmological constant boundary are called quintom models [14], and there are a lot of interests in the phenomenological study of this class of models recently in the literature $13,15,16,17,18,19$, $20,21,22,23]$.

2 We take the convention for the sign of the metric with $(+,-,-,-)$.
} 
where $M$ is a constant with mass dimension and $c$ is a dimensionless constant. One can see that this model differs from the phantom model [1] due to the dimension- 6 operator. Our analysis below will show that this model gives rise to an equation of state which transit from $w>-1$ to $w<-1$ as the redshift decreases, furthermore it is equivalent to the two scalar fields model of the quintom dark energy studied in Ref. 14, 17, 23].

Given the Lagrangian (2) we have the energymomentum tensor:

$$
\begin{aligned}
T^{\mu \nu}= & {\left[\frac{1}{2} \nabla_{\rho} \phi \nabla^{\rho} \phi+\frac{c}{2 M^{2}} \square \phi \square \phi+\frac{c}{M^{2}} \nabla^{\rho} \phi \nabla_{\rho}(\square \phi)\right.} \\
& +V] g^{\mu \nu}-\nabla^{\mu} \phi \nabla^{\nu} \phi-\frac{c}{M^{2}} \nabla^{\nu} \phi \nabla^{\mu}(\square \phi) \\
& -\frac{c}{M^{2}} \nabla^{\mu} \phi \nabla^{\nu}(\square \phi),
\end{aligned}
$$

and the equation of motion:

$$
-\square \phi-\frac{c}{M^{2}} \square^{2} \phi+\frac{d V}{d \phi}=0 .
$$

The energy-momentum tensor (3) can be rewritten as

$$
\begin{aligned}
T^{\mu \nu}= & \frac{1}{2}\left[\nabla_{\rho} \psi \nabla^{\rho} \psi-\nabla_{\rho} \chi \nabla^{\rho} \chi+2 V(\psi-\chi)+\right. \\
& \left.\frac{M^{2}}{c} \chi^{2}\right] g^{\mu \nu}-\nabla^{\mu} \psi \nabla^{\nu} \psi+\nabla^{\mu} \chi \nabla^{\nu} \chi
\end{aligned}
$$

where $\chi$ and $\psi$ are defined by

$$
\begin{aligned}
& \chi=\frac{c}{M^{2}} \square \phi, \\
& \psi=\phi+\chi .
\end{aligned}
$$

It is not difficult to see that the energy-momentum tensor (5) can be derived from the following Lagrangian

$$
\mathcal{L}=-\frac{1}{2} \nabla_{\mu} \psi \nabla^{\mu} \psi+\frac{1}{2} \nabla_{\mu} \chi \nabla^{\mu} \chi-V(\psi-\chi)-\frac{M^{2}}{2 c} \chi^{2},
$$

with $\psi$ and $\chi$ being two independent fields. The variations of the Lagrangian in (8) with respect to the fields $\psi$ and $\chi$ respectively give rise to the following equations of motions,

$$
\begin{aligned}
& \square \psi-V^{\prime}=0, \\
& \square \chi+\frac{M^{2}}{c} \chi-V^{\prime}=0,
\end{aligned}
$$

where the prime denotes the derivative with respect to $\psi-\chi$.

When we take $\psi=\phi+\chi$ in (8) and (9), we recover the equation of motion (4) of the single field model and the transformation equation (6). So, one can see from the derivations above that our single field model (2) is equivalent to the one with two fields (8) where one is the normal (quintessence-like) scalar field $\chi$ and another is the ghost (phantom-like) field $\psi$. In general, the normal mode $\chi$ gives rise to the equation of state $w_{1} \geq-1$ and the ghost mode has $w_{2} \leq-1$, consequently the energy density in $\chi$ would decrease and that in $\psi$ would increase with the expansion of the universe. Assuming $\chi$ dominates the dark energy sector in the early time, the equation of state for the system will be larger than -1 during that period of time. When the ghost mode becomes dominant over the normal one (and will continue to be dominant among all the components in the universe), the equation of state of the system will become $<-1$ and cross the cosmological constant boundary at a intermediate redshift. Quantitatively the moment when the equation of state crosses -1 depends on the values of the model parameters.

Now we study the perturbations of our model. As is well known, in the usual quintessence and phantom models, the field and metric perturbations are stable on the small length scales. For both the quintessence and the phantom models the sound speed as indicated in Ref. 30] is given by

$$
c_{s}^{2}=\frac{\partial p / \partial X}{\partial \rho / \partial X}=1
$$

where $X \equiv(1 / 2) \nabla_{\mu} \phi \nabla^{\mu} \phi$. This can be seen clearly from the dispersion relations. For the phantom field, the frequency $\omega$ relates to the momentum $\mathbf{k}$ in the same way as in the case of the quintessence, $\omega^{2}=\mathbf{k}^{2}+\ldots$. And the sound speed is often defined as $\omega^{2}=c_{s}^{2} \mathbf{k}^{2}+\ldots$. Since we have demonstrated that our model is equivalent to a model with a quintessence-like and a phantom-like scalar fields, the general arguments for the quintessence and the phantom hold here, which indicate there will be no exponentially instabilities of the perturbations on small scales in our model.

In general the potential term $V(\psi-\chi)$ (equivalently $V(\phi)$ in our single field model) should include the interactions between the two fields $\psi$ and $\chi$. For some specific choices of the potential $V$, however these two modes could decouple. As an example we consider $V=(1 / 2) m^{2} \phi^{2}$. Then the Lagrangian (8) can be "diagonalized" as

$$
\mathcal{L}=\frac{1}{2} \nabla_{\mu} \phi_{1} \nabla^{\mu} \phi_{1}-\frac{1}{2} \nabla_{\mu} \phi_{2} \nabla^{\mu} \phi_{2}-\frac{1}{2} m_{1}^{2} \phi_{1}^{2}-\frac{1}{2} m_{2}^{2} \phi_{2}^{2},
$$

through the transformation

$$
\left(\begin{array}{l}
\psi \\
\chi
\end{array}\right)=\left(\begin{array}{ll}
-a_{1} & a_{2} \\
-a_{2} & a_{1}
\end{array}\right)\left(\begin{array}{l}
\phi_{1} \\
\phi_{2}
\end{array}\right) .
$$

In (12) and (13)

$$
\begin{aligned}
& a_{1}=\frac{1}{2}\left(1+\frac{4 c m^{2}}{M^{2}}\right)^{-1 / 4}\left(\sqrt{1+\frac{4 c m^{2}}{M^{2}}}-1\right), \\
& a_{2}=\frac{1}{2}\left(1+\frac{4 c m^{2}}{M^{2}}\right)^{-1 / 4}\left(\sqrt{1+\frac{4 c m^{2}}{M^{2}}}+1\right),
\end{aligned}
$$

and

$$
\begin{aligned}
& m_{1}^{2}=\frac{M^{2}}{2 c}\left(\sqrt{1+\frac{4 c m^{2}}{M^{2}}}+1\right), \\
& m_{2}^{2}=\frac{M^{2}}{2 c}\left(\sqrt{1+\frac{4 c m^{2}}{M^{2}}}-1\right) .
\end{aligned}
$$


Hence, one can see that our model (2) with $V=$ $(1 / 2) m^{2} \phi^{2}$ is equivalent to the uncoupled system (12). The two modes $\phi_{1}$ and $\phi_{2}$ evolve independently in the universe. The positivity of the parameter $c$ guarantees the positivity of $m_{1}^{2}$ ( $m_{2}^{2}$ is always positive as long as $\left.1+\frac{4 c m^{2}}{M^{2}}>0\right)$. In the limit of $m \ll M$, the masses of the two modes are approximately $m_{1} \simeq M / \sqrt{c}$ and $m_{2} \simeq m$. In fact, $\phi_{1}$ and $\phi_{2}$ are the eigenfunctions of the d'Alembertian operator, $\square$, with the eigenvalues $-m_{1}^{2}$ and $m_{2}^{2}$ respectively. The solution to the equation of motion (4) $\phi$ is decomposed by these eigenfunctions as $\phi=\left(1+\frac{4 c m^{2}}{M^{2}}\right)^{-1 / 4}\left(\phi_{1}+\phi_{2}\right)$.

As we mensioned above, the perturbations in the phantom component $\phi_{2}$ present no unphysical instabilities in the classical level. In fact, the spatial fluctuation of $\phi_{2}$ with wavenumber $k>m_{2}$ is stable, the solution to the equation of motion is oscillatory with time. The time-rising behavior only presents on very large scales, $L>1 / m_{2}$. If the phantom mass $m_{2}$ of the phantom field is smaller than $H$, there will be no instability within the horizon 31]. The rising behaviors of the super-horizon modes (including the background mode, which corresponds to $k=0$ ) of phantom do not matter in cosmology. This is because the universe is expanding instead of static, generally the components in the universe are evolving. Furthermore, the Hubble expansion provides a friction force which prevents these modes from increasing exponentially. In another word, such an instability is "benign" 32.

In Fig 1 we plot the equation of state of our model as a function of $\ln a$. One can see clearly $w$ crosses -1 during evolution. Furthermore, we made a test on the equivalence of the model (2) with the two-field quintom model numerically for $V=(1 / 2) m^{2} \phi^{2}$. If the background spacetime is described by the FriedmannRobertson-Walker metric, $d s^{2}=d t^{2}-a^{2}(t) \delta_{i j} d x^{i} d x^{j}$ and the field $\phi$ is homogeneous, the d'Alembertian operator will depend only on the time derivatives, $\square=$ $\partial^{2} / \partial t^{2}+3 H \partial / \partial t$. The equation of motion (41) becomes:

$$
-\ddot{\phi}-3 H \dot{\phi}+m^{2} \phi-\frac{c}{M^{2}}\left(\frac{\partial^{2}}{\partial t^{2}}+3 H \frac{\partial}{\partial t}\right)(\ddot{\phi}+3 H \dot{\phi})=0,
$$

where dot represents the derivative with respect to time. The energy density and the pressure are

$\rho=-\frac{1}{2} \dot{\phi}^{2}+\frac{c}{2 M^{2}}(\ddot{\phi}+3 H \dot{\phi})^{2}-\frac{c}{M^{2}} \dot{\phi} \frac{\partial}{\partial t}(\ddot{\phi}+3 H \dot{\phi})+\frac{1}{2} m^{2} \phi^{2} ;$

$p=-\frac{1}{2} \dot{\phi}^{2}-\frac{c}{2 M^{2}}(\ddot{\phi}+3 H \dot{\phi})^{2}-\frac{c}{M^{2}} \dot{\phi} \frac{\partial}{\partial t}(\ddot{\phi}+3 H \dot{\phi})-\frac{1}{2} m^{2} \phi^{2}$,

respectively. The initial conditions picked up in the numerically calculations are

$$
\begin{aligned}
\phi(0) & =g\left[\phi_{1}(0)+\phi_{2}(0)\right], \\
\dot{\phi}(0) & =g\left[\dot{\phi}_{1}(0)+\dot{\phi}_{2}(0)\right], \\
\ddot{\phi}(0) & =g\left[-3 H(0)\left(\dot{\phi}_{1}(0)+\dot{\phi}_{2}(0)\right)-m_{1}^{2} \phi_{1}(0)+m_{2}^{2} \phi_{2}(0)\right],
\end{aligned}
$$

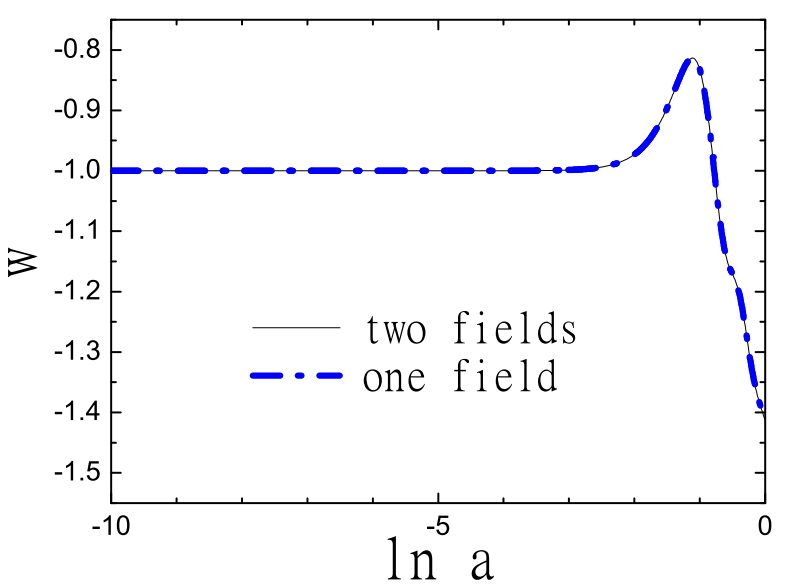

FIG. 1: Plots of the equation of state for our model and the two-field model with one quintessence and one phantom field. In the numerical calculations we choose the initial conditions given by Eq. (19) and have set $m=2 \times 10^{-61} M_{p l}, \frac{c}{M^{2}}=$ $10^{120} M_{p l}^{-2}$. The equivalent initial conditions for the two scalar fields are $\phi_{1}(0)=3 \times 10^{-2} M_{p l}, \phi_{2}(0)=0.18 M_{p l}$, and $\dot{\phi}_{1}(0)=$ $0, \dot{\phi}_{2}(0)=0 . \Omega_{D E} \approx 0.73$ and $h \approx 0.68$.

$$
\begin{aligned}
\dddot{\phi}(0)= & g\left[\left(9 H(0)^{2}-3 \dot{H}(0)\right)\left(\dot{\phi}_{1}(0)+\dot{\phi}_{2}(0)\right)+\right. \\
& 3 H(0)\left(m_{1}^{2} \phi_{1}(0)-m_{2}^{2} \phi_{2}(0)\right)- \\
& \left.\left(m_{1}^{2} \dot{\phi}_{1}(0)-m_{2}^{2} \dot{\phi}_{2}(0)\right)\right] \\
H^{2}(0)= & \frac{8 \pi G}{3}\left[\rho_{m}(0)+\rho_{r}(0)+\frac{1}{2} \dot{\phi}_{1}^{2}(0)-\frac{1}{2} \dot{\phi}_{2}^{2}(0)+\right. \\
& \left.\frac{1}{2} m_{1}^{2} \phi_{1}^{2}(0)+\frac{1}{2} m_{2}^{2} \phi_{2}^{2}(0)\right], \\
\dot{H}(0)= & -4 \pi G\left[\rho_{m}(0)+\frac{4}{3} \rho_{r}(0)+\dot{\phi}_{1}^{2}(0)-\dot{\phi}_{2}^{2}(0)\right],
\end{aligned}
$$

where 0 in the bracket means the initial time, $\rho_{m}$ and $\rho_{r}$ are the energy densities of matter and radiation respectively, and $g$ is defined as

$$
g \equiv\left(1+\frac{4 c m^{2}}{M^{2}}\right)^{-1 / 4} .
$$

We depicte the numerical result for the comparison in Fig (1) One can see from this figure the equation of state from (17) and (18) of our single scalar field model does coincide with that of the two-field model (12).

In summary, we in this paper have proposed a single scalar field model of dark energy which has the property of crossing the cosmological constant boundary. This is achieved by considering higher derivative operators. And this class of model is free from the difficulties of the singularity and the gravitational instabilities in the general k-essence-like models. We should point out that although the energy-momentum tensor of this single scalar field 
model is equivalent to two independent scalar fields ${ }^{3}$, when we consider the interactions they may possibly show some different behaviors. It might be theoretically possible to solve the inherent problems, the quantum instabilities exhibited in the model of phantom [34] and is worth studying further.

Our study here has the following implications: 1) in the general phenomenological fitting appearing in the literature of the dark energy models to the observational data such as SN, CMB, LSS an issue is how to consistently include the perturbations of the dark energy. The key to this issue is to build theoretical models of dark energy with the equation of state crossing -1 . Our model proposed in this paper serves as an example for this class of models of dark energy to study the cosmological im-

3 After our paper appeared, the authors of Ref. 33] used the " $\square$ " term only and dropped the conventional kinetic term as well the plications numerically; 2) similar to the k-inflation our model can be applied for inflation. And in this case one would expect non-vanishing isocurvature perturbations even though this model includes explicitly only one inflaton field.

Acknowledgements: M.L. would like to thank Michael Doran and Christof Wetterich for discussions and to acknowledge the support from the Alexander von Humboldt Foundation. B.F. and X.Z. would like to thank Xiao-Jun Bi, Hong Li and Yun-Song Piao for discussions and are supported in part by the National Natural Science Foundation of China under the grant No. 90303004 and also by the Ministry of Science and Technology of China under grant No. NKBRSF G19990754.

potential, which cannot be identified with two scalar fields.
[1] Riess A G et al., 1998 Astron. J. 1161009 astro-ph/9805201

[2] Perlmutter S et al., 1999 Astrophys. J. 517565 astro-ph/9812133

[3] Spergel D N et al., 2003 Astrophys. J. Suppl. 148175 astro-ph/0302209

[4] Seljak U et al., 2005 Phys. Rev. D $71 \quad 103515$ astro-ph/0407372

[5] Alam U, Sahni V and Starobinsky A A, 2004 JCAP 0406 008 astro-ph/0403687; Huterer D and Cooray A, 2005 Phys. Rev. D 71023506 astro-ph/0404062; Gong Y, 2005 Class. Quant. Grav. 222121 astro-ph/0405446

[6] Corasaniti P S, Kunz M, Parkinson D, Copeland E $\mathrm{J}$ and Bassett B A, 2004 Phys. Rev. D $\mathbf{7 0} 083006$ astro-ph/0406608; Hannestad S and Mortsell E, 2004 JCAP 0409001 astro-ph/0407259; Upadhye A, Ishak $\mathrm{M}$ and Steinhardt P J, 2005 Phys. Rev. D 72063501 astro-ph/0411803

[7] Wetterich C, 1988 Nucl. Phys. B 302668

[8] Wetterich C, 1995 Astron. Astrophys. 301321 hep-th/9408025

[9] Ratra B and Peebles P J E, 1988 Phys. Rev. D 373406

[10] Caldwell R R, Dave R and Steinhardt P J, 1998 Phys. Rev. Lett. 801582 astro-ph/9708069

[11] Caldwell R R, 2002 Phys. Lett. B 54523 astro-ph/9908168

[12] Zhao G-B, Xia J -Q, Li M, Feng B and Zhang X, 2005 Preprint astro-ph/0507482

[13] Vikman A, 2005 Phys. Rev. D $\mathbf{7 1} 023515$ astro-ph/0407107

[14] Feng F, Wang X and Zhang X, 2005 Phys. Lett. B 60735 astro-ph/0404224; Feng B, Li M, Piao Y -S and Zhang X, 2004 Preprint astro-ph/0407432

[15] Wei Y -H and Tian Y, 2004 Class. Quant. Grav. 215347 gr-qc/0405038

[16] Zhang X, 2004 Preprint hep-ph/0410292

[17] Guo Z -K, Piao Y -S, Zhang X and Zhang Y -Z, 2005 Phys. Lett. B 608177 astro-ph/0410654

[18] Hu W, 2005 Phys. Rev. D 71047301 astro-ph/0410680
[19] Xia J -Q, Feng B and Zhang X, 2005 Mod. Phys. Lett. A 202409 astro-ph/0411501

[20] Caldwell R R and Doran M, 2005 Phys. Rev. D 72043527 astro-ph/0501104

[21] Li B J, Chu M -C, Cheung K C and Tang A, 2005 Preprint astro-ph/0501367

[22] Wei H and Cai R-G, 2005 Class. Quant. Grav. 223189 hep-th/0501160

[23] Zhang X F, Li H, Piao Y -S and Zhang X, 2005 Preprint astro-ph/0501652

[24] Simon J Z, 1990 Phys. Rev. D 413720

[25] Elizer A and Woodard R P, 1989 Nucl. Phys. B 325389

[26] Erler T G and Gross D J, Preprint hep-th/0406199

[27] Stelle K S, 1977 Phys. Rev. D 16953

[28] Arkani-Hamed N, Cheng H -C, Luty M A and Mukohyama S, 2004 JHEP 0405074 hep-th/0312099; ArkaniHamed N, Creminelli P, Mukohyama S and Zaldarriaga M, 2004 JCAP 0404001 hep-th/0312100; Anisimov A and Vikman A, 2005 JCAP 0504009 hep-ph/0411089; Mukohyama S, 2005 Phys. Rev. D 71 104019 hep-th/0502189; Arkani-Hamed N, Cheng H -C, Luty M A, Mukohyama S and Wiseman T, 2005 Preprint hep-ph/0507120

[29] Ostrogradski M, 1850 Mem. Ac. St. Petersbourg VI 4385

[30] Garriga J and Mukhanov V F, 1999 Phys. Lett. B 458 219 hep-th/9904176

[31] Hsu S D H, Jenkins A and Wise M B, 2004 Phys. Lett. B 597270 astro-ph/0406043; Buniy R V and Hsu S D H, 2005 Preprint hep-th/0502203

[32] Smilga A V, 2005 Nucl. Phys. B $706 \quad 598$ hep-th/0407231; 2005 Preprint hep-th/0503213

[33] Anisimov A, Babichev E and Vikman A, 2005 JCAP 0506 006 astro-ph/0504560

[34] Carroll S M, Hoffman M and Trodden M, 2003 Phys. Rev. D 68023509 astro-ph/0301273; Cline J M, Jeon S -Y and Moore G D, 2004 Phys. Rev. D 70043543 hep-ph/0311312 